ИЗВЕСТИЯ АКАДЕМИИ НАУК ЭСТОНСКОИ ССР. ФИЗИКА МАТЕМАТИКА

PROCEEDINGS OF THE ACADEMY OF SCIENCES OF THE ESTONIAN SSR.

PHYSICS * MATHEMATICS

$1987,36,3$

удК 528.88

A. МАРШАK

\title{
О ВОССТАНОВЛЕНИИ ОПТИЧЕСКИХ. ПАРАМЕТРОВ ПОСЕВА ПО КОЭФФИЦИЕНТУ СПЕКТРАЛЬНОЙ ЯРКОСТИ МЕТОДОМ МОНТЕ-КАРЛО
}

\author{
(Представил Я. Эйнасто)
}

\section{1. Введение}

В последние годы в связи с бурным развитием аэрокосмического зондирования растительности возросла потребность в решении обратных задач зондирования, т. е. в восстановлении оптических и геометрических параметров растительного покрова (РП) по данным о спектральной яркости такого покрова.

Такая задача для модели Суйтса $\left[{ }^{1}\right]$, в которой радиационный режим РП описывался при помощи системы дифференциальных уравнений, была поставлена и частично решена в $\left[{ }^{2,3}\right]$. В дальнейшем $\left[{ }^{4,5}\right]$ удалось обратить и другие модели РП, такие как модели Бунника $\left[{ }^{6}\right]$ и Нормана $\left[{ }^{7}\right]$. Сравнивая эти модели с другими моделями, (см. например $\left[{ }^{8}\right]$ и $\left.\left[{ }^{9}\right]\right)$ можно заключить, что одни из них слишком сложны и исчерпывающи, но неудобны для практической реализации, а другие, хотя и просты и понятны, гораздо менее реалистичны.

В данной работе предлагается аппроксимация посева при помощи случайно и независимо распределенных фитоэлементов. Коэффициент спектральной яркости (КСЯ) такого РП при заданных условиях освещения рассчитывается методом Монте-Карло (см. $\left.\left[{ }^{10-12}\right]\right)$. Целью настоящей работы является обращение такой модели, а именно, восстановление и оценка оптических свойств фитоэлементов и почвы.

\section{2. Модель посейа}

B качестве первого приближения РП моделировался при помощи круглых горизонтальных матовых листьев, центры которых равномерно распределены в параллелепипедах, определяемых средним расстоянием между листьями по вертикали и горизонтали. Архитектурные параметры посева задавались следующими четырьмя величинами: $L, d$, $N$ и p. Здесь $L-$ индекс листовой поверхности, $d-$ диаметр листа, $N$ - число листьев по вертикали, а $p$ - характеристика угловых размеров листа (отношение расстояния между листьями по вертикали к диаметру листа).

Оптические параметры листьев и почвы определялись спектральными коэффициентами отражения $r$ и пропускания $t$ листьев и КСЯ почвы @. (Это те параметры, восстановлению которых по данным об отражении от РП и посвящена настоящая работа.)

Вопросы обобщения модели на РП с заданным конкретным расположением листьев и распределением их угловой нормали, а также па РП со стеблями и другими фитоэлементами рассматриваются ниже. 


\section{3. Метод решения прямой задачи}

Радиационный режим такого РП рассчитывался методом МонтеКарло. Опишем кратко алгоритм расчета для данной модели.

Пусть $\xi^{i}$ - случайная величина, независимые реализации которой представимы в виде

$$
\xi^{i, j}=\sum_{k=0}^{l_{f}} Q^{i, j}\left(x_{k}\right), \quad j=1,2, \ldots, N_{\Phi}, \quad i=1,2, \ldots, M .
$$

Здесь $x_{0}, x_{1}, \ldots, x_{l}$ - однородная цепь Маркова, соответствующая точкам траектории каждого фотона, $l_{j}$ - номер последнего учитываемого столкновения, а $N_{\phi}$ - число пропускаемых фотонов. Величина

$$
Q^{i, j}\left(x_{k}\right)=B_{j}\left(x_{k}\right) P_{i}\left(x_{k}\right),
$$

где $B_{j}\left(x_{k}\right)$ - «вес» фотона в точке $x_{k}$ для $j$-й траектории

$$
B_{j}\left(x_{0}\right)=1, \quad B_{j}\left(x_{k}\right)= \begin{cases}(r+t) B_{j}\left(x_{k-1}\right), & x_{k} \in L, \\ \varrho B_{j}\left(x_{k-1}\right), & x_{k} \in S\end{cases}
$$

(здесь $L-$ поверхность листа, а $S$ - поверхность почвы),

$P_{i}\left(x_{h}\right)=\left\{\begin{array}{l}\sigma \int g(\gamma) d \omega \\ \Omega_{t} \\ 0\end{array}\right.$

$$
\begin{aligned}
& \text { если фотон из точки } x_{k} \text { РП мо- } \\
& \text { жет вылететь в направлении } \\
& i \text {-го приемника в телесном уг- } \\
& \text { ле } \Omega_{i} \text {, } \\
& \text { в противном случае. }
\end{aligned}
$$

Величина б есть $r, t$ или е в зависимости от столкновения, $g$ - индикатриса рассеяния фитоэлементов или почвы.

Таким образом, $Q^{i, j}\left(x_{k}\right)$ есть вклад $j$-го фотона, находящегося в точке $x_{k}$, в оценку $i$-го функционала. Вычисляя срднее арифметическое $N_{\text {ф }}$ реализаций, мы получаем смещенную оценку для $i$-го функционала, а именно:

$$
F_{i} \approx M \xi^{i} \approx\left(1 / N_{\phi}\right) \sum_{j=1}^{N_{\phi}} \xi^{i, j}
$$

есть вероятность того, что падающий на РП в направлении $(\vartheta, \varphi)$ фотон отражается обратно в телесном угле $\Omega_{i}=\Delta \theta_{i} \Delta \varphi, i=1, \ldots, L$ в направлении $\left(\theta_{i}, \varphi\right)$. (Здесь $M$ обозначает математическое ожидание.)

Более подробно алгоритм метода Монте-Карло описан в [ $\left.{ }^{10}\right]$. Там же приводится оценка точности этого метода и рассматривается метод расщепления, при помощи которого находится оптимальное число траекторий, которое пропускается при одном значении случайной величины, характеризующей случайность построения модели РП.

\section{4. Решение и особенности обратной задачи}

Рассмотрим обратную задачу определения параметра $\sigma$ по известным значениям интенсивностей $I_{k}\left[{ }^{13}\right]$, т. е. рассмотрим систему нелинейных уравнений

$$
F_{k}\left(\sigma_{1}, \sigma_{2}, \ldots, \sigma_{m}\right)=I_{k}, \quad k=1,2, \ldots, M,
$$

или в операторной форме

$$
F(\sigma)=I
$$

Уравнение (5) будем решать методом Ньютона-Канторовича [ $\left.{ }^{14}\right]$ 


$$
\sigma_{i}^{i+1}=\sigma_{i}^{l}+\Delta \sigma_{i}^{l}, \quad i=1,2, \ldots, m, \quad l=0,1, \ldots,
$$

где $\Delta \sigma_{i}{ }^{l}$ удовлетворяет системе

$$
\sum_{i=1}^{m} \frac{\partial F_{k}}{\partial \sigma_{i}} \Delta \sigma_{i}^{l}=I_{k}-F_{k}\left(\sigma_{1}^{l}, \sigma_{2}^{l}, \ldots, \sigma_{m}^{l}\right), \quad k=1,2, \ldots, M .
$$

В итерационном процессе (6) за начальное приближение принимается некоторый $\sigma^{0}$, элементы которого представляют прогностические значения искомых параметров. Остановку процесса (6) будем производить в случае, если

$$
\left|I_{k}-F_{k}\left(\sigma^{l}\right)\right| \leqslant \varepsilon,
$$

где $\varepsilon>0-$ заданное малое число.

Обозначая матрицу системы (7) через $A$, а свободный член через $g$, получим

$$
A x=g,
$$

где $x$ значение вектора $\Delta \sigma$ на $l$-м шаге итерации. Вместо системы (9) мы решаем регуляризованную систему

$$
\left(\alpha E+A^{\prime} A\right) x=A^{\prime} g,
$$

где $A^{\prime}$ - транспонированная матрица. Малый положительный параметр $\alpha$ выбирался из регуляризационных соображений и его величина зависела от обусловленности матрицы $A^{\prime} A$ (во всех численных расчетах эта матрица была очень плохо обусловлена: ее число обусловленности имело порядок $\left.10^{3}-10^{5}\right)$.

Вычисление матрицы $A$ проводилось методом Монте-Карло по тем же траекториям, что при вычислении функционалов $F_{k}, k=1,2, \ldots, M$. В предположении о возможности дифференцирования под знаком математического ожидания $\left[{ }^{15}\right]$, из (1) и (4) получим

$$
\begin{gathered}
\frac{\partial F_{i}}{\partial \sigma_{p}} \approx M \sum_{k=0}^{l_{l}} \frac{\partial Q^{i, j}\left(x_{k}, \sigma\right)}{\partial \sigma_{p}}= \\
=M \sum_{k=0}^{l_{l}} Q^{i, j}\left(x_{k}, \sigma\right) \cdot\left[\frac{\partial \ln P_{i}}{\partial \sigma_{p}}+\frac{\partial \ln B_{j}}{\partial \sigma_{p}}\right], \quad p=1,2, \ldots, m .
\end{gathered}
$$

Последние производные легко вычисляются по формулам (2)-(3), где $m=3, \sigma_{1}=r, \sigma_{2}=t, \sigma_{3}=\mathrm{Q}$.

\section{5. Численные эксперименты}

Важным в решении обратных задач имеет выбор направлений визирования, т. е. тех направлений, по которым снимаются начальные данные $I_{k}$ и оцениваются функционалы $F_{k}, k=1,2, \ldots, M$ (см. (5)). Следуя $\left[{ }^{11}\right]$ и $\left[{ }^{12}\right]$, наиболее информативными областями, т. е. наиболее чувствительными к вариации восстанавливаемых параметров, являются по азимуту - плоскость вертикала солнца, а по широте - околонадирные области и область вокруг обратного блеска.

Были проведены серии расчетов по восстановлению оптических параметров посева и яркости почвы в двух спектральных областях, соответствующих фотосинтетически активной (ФАР) и близкой инфракрасной (БИКР) радиациям. Отметим, что если для области ФАР при решении прямой задачи мы могли ограничиться учетом лишь двукратно рассеянной радиации, то в области БИКР приходилось рассчитывать траектории фотонов до $6-7$ столкновений. 
Посев аппроксимировался описанной выше моделью, в которой варьировались значения архитектурных параметров $L, d, N$ и $p$.

Итерационный процесс проводили следующим образом: искомые оптические параметры возмущали на $50-200 \%$ и полученные значения брали за начальные приближения. В результате решения обратной задачи коэффициент отражения листа $r$ восстанавливался с точностью $5 \%$ за $1-3$ итерации, коэффициент пропускания $t$ за такое же число итераций восстанавливался с точностью порядка $10 \%$, а восстановление яркости почвы сильно зависело от плотности посева $L$ : при больших $L$ итерационный процесс для оценки @ вообще не сходился, а при малых $(L=1-2)$ - сходился относительно неплохо. (Это и естественно, ибо в оптически толстых посевах почвы практически не видно и восстановить ее яркость с достаточной точностью по данным об отражении от РП не представляется возможным (на это, кстати, указывается и в $\left.\left[{ }^{3-5}\right]\right)$.) Худшее восстановление коэффициента пропускания объясняется горизонтальностью листьев, откуда следует, что производная по этому параметру от фотонов первой кратности равна нулю.

Отметим, что в системе (9) значения матрицы $A$ и вектора $g$ даны приближенно. Точность $g$ соответствует точности решения прямой задачи и в нашем случае погрешность не превышала $5 \%$. Хуже обстояло дело с матрицей производных $A$. В посевах средней оптической толщины $(L=2-3)$ она определялась с точностью $20-40 \%$ и $A^{\prime} A$ была очень плохо обусловлена. Из сказанного выше следует, что в правиле остановки (8) итерационного процесса (6) нельзя выбирать $\varepsilon$ слишком малым, ибо в противном случае при попадании в окрестность решения восстановленные параметры начинают портиться и дальнейшие итерации лишь ухудшают их значения. Полученные оптические параметры можно использовать как начальные приближения для более точных методов.

Заметим, что при фиксировании коэффициента яркости почвы описанный процесс сходится значительно лучше.

\section{6. Заключение}

В данной работе по методике решения обратных задач при помощи метода Монте-Карло [ $\left.{ }^{15}\right]$ восстанавливались оптические параметры посева, модель которого описана в $\left[{ }^{10}\right]$. Надо заметить, что методика, развитая для решения задач атмосферной оптики, в которых процесс переноса радиации довольно хорошо описывается кинетическим уравнением переноса, может быть применена и к средам, где уравнение переноса не работает или работает плохо. К таким средам относится и РП.

Обобщение полученных результатов на более реальные посевы не представляет особого труда. Для этого надо уметь решать достаточно точно и быстро (в смысле времени работы ЭВМ) прямые задачи. Наличие листьев произвольной ориентации лишь улучшает решение обратной задачи, ибо в этом случае в приемники, стоящие над РП, попадает излучение, отраженное от обеих сторон листа и поэтому производные от коэффициента пропускания будут вычисляться более точно. Прибавление к модели посева моделей стеблей, колосьев, цветков и других фитоэлементов лишь усложняет решение прямой задачи, а на сам итерационный процесс оказывает мало влияния.

Хуже обстоит дело с восстановлением архитектурных параметров посева, определение которых, несомненно, представляет наибольший интерес. Особенно это касается оценки индекса листовой поверхности $L$ и распределения угловых нормалей листа, так как эти параметры наиболее трудно измерить в реальных РП. Приведенная выше известная методика не распространяется на восстановление этих параметров, ибо 
при оценке функционалов методом Монте-Карло величины $Q$ (см. (1)) явно не выражаются через геометрические параметры (т. е. для них отсутствуют формулы связи типа (2)-(3)), и на той же самой траектории брать производные по этим параметрам нельзя. По-видимому, здесь можно предложить модификацию метода Ньютона-Канторовича, по которой производные берутся ни в каждой точке, а лишь в одной, скажем, начальной. Вычисляя предварительно эти производные, можно построить сходящийся итерационный процесс. Однако вопросы сходимости и области сходимости такого процесса для данной модели подлежат тщательному исследованию.

Л И ТЕРА Т У Р А

1. Suits, G. H. Remote Sens. Environ., 2, 117-125 (1972).

2. Goel, N. S., Strebel, D. E., Thompson, R. L. Remote Sens. Environ., № 14, 77-111 (1984).

3. Goel, N. S., Thompson, R. L. Remote Sens. Environ., № 15, 223-236 (1984).

4. Goel, N. S., Thompson, R. L. Remote Sens. Environ., № 15, 237-253 (1984).

5. Goel, N. S., Thompson, R. L. Remote Sens. Environ., № 16, 69-85 (1984).

6. Bunnik, N.J.J. The Multispectral Reflectance of Shortwave Radiation by Agricultural Crops in Relation with their Morphological and Optical Properties. Mededelingen Landbouwhogescholl Wageningen, Nederland, № 78-1, 1978.

7. Norman, J. M. In: Heat and Mass Transfer in the Biosphere. Part I. Transfer Processes in the Plant Environment. Washington-New York-LondonSydney-Toronto, J. Wiley \& Sons, 1975, 187-205.

8. Росс Ю. К. Радиационный режим и архитектоника растительного покрова. Л, Гидрометеоиздат, 1975.

9. Нильсон Т., Кууск А. Исслед. Земли из космоса, № 5, 76-83 (1984).

10. Росс Ю. К., Маршак А. Л. Исслед. Земли из космоса, № 5, 58-67 (1984).

11. Росс Ю. К., Мариак А. Л. Исслед. Земли из космоса, № 2, 96-105 (1987).

12. Росс Ю. К., Мариак А. Л. Исслед. Земли из космоса, № 2, 86-93 (1987).

13. Марчук Г. Н. Космич. исследования, 2, № 3, 462-477 (1964).

14. Канторович Л. В., Акилов Г. П. Функциональный анализ. М., «Наука», 1979

15. Ермаков С. М., Михайлов Г. А. Курс статистического моделирования. М., «Наука», 1976.

Институт астрофизики и физики атмосферы Академии наук Эстонской ССР
Поступила в редакцию $30 /$ IX 1986

\section{A. MARSAK}

\section{TAIMKATTE OPTILISTE PARAMEETRITE TAASTAMISEST SPEKTRAALSE HELEDUSKOEFITSIENDI JÄRGI MONTE CARLO MEETODIL}

On kirjeldatud pöördülesannete lahendamise üldist metoodikat taimkatte parameetrite taastamiseks Monte Carlo meetodil. Taimkatet modelleeritakse horisontaalsete mattide lehtedega. On toodud arvuliste eksperimentide tulemused.

\section{A. MARSHAK}

\section{ON THE RESTITUTION OF THE OPTICAL PARAMETERS OF THE CANOPY, USING THE DATA OF SPECTRAL BRIGHTNESS COEFFICIENT BY THE MONTE CARLO METHOD}

The general technique of the solution of the inverse problem for estimating canopy parameters by the Monte Carlo method is described. The canopy is simulated by the horizontal dull leaves. The results of numerical experiments for estimating coefficients of transmittance and reflectance of leaves and the brightness coefficient of soil are given. The inversion of canopy reflectance model for estimating leaf area index is discussed. 